\title{
COMPACT GROUPS OF REAL POWER NEED NOT BE METRIZABLE
}

\author{
DONALD HACKLER
}

We show that the metrizability of compact (separable Abelian) groups of power $c=2^{\omega}$ can be neither proved nor disproved in ZFC. The local weight of a topological group is the least power of a basis at the identity (in a locally compact group, the least power of an open family whose intersection is the identity). It is known that a topological group is metrizable just in case it has local weight $\omega[\mathbf{K}]$.

One can show that a (locally) compact group of power $\kappa$ has local weight $<\kappa$ (for $\kappa=\omega_{1}$ this is $[\mathbf{J}]$ ). Hence the continuum hypothesis implies that each (locally) compact group of power $c$ is metrizable.

On the other hand, it is consistent that there be compact groups of power $c$ and uncountable local weight. In fact, it is consistent to assume Martin's Axiom plus $c>\omega_{1}$, and Martin's Axiom implies that $2^{\kappa}=c$ whenever $\omega \leqslant \kappa$ $<c$ (see [ST] and [MS]). For each such $\kappa, G=Z_{2}^{\kappa}$ is a compact separable Abelian group of power $2^{\kappa}=c . G$ is not metrizable, since $0=(0,0,0, \ldots)$ is not the intersection of $<\kappa$ basic open sets.

\section{REFERENCES}

[J] F. B. Jones, On the first countability axiom for locally compact Hausdorff spaces, Colloq. Math. 7 (1959), 33-34. MR 22 \#2972.

[K] S. Kakutani, Über die Matrisation der topologischen Gruppen, Proc. Imper. Acad. Japan 12 (1936), 82-84.

[MS] D. Martin and R. Solovay, Internal Cohen extensions, Ann. Math. Logic 2 (1970), no. 2, 143-178. MR 42 \# 5787.

[ST] R. M. Solovay and S. Tennenbaum, Iterated Cohen extensions and Souslin's problem, Ann. of Math. (2) 94 (1971), 201-245. MR 45 \#3212.

Department of Mathematics, University of Wisconsin, Madison, Wisconsin 53706

Received by the editors February 24, 1976 and, in revised form, June 7, 1976.

AMS (MOS) subject classifications (1970). Primary 54A25, 54E35, $22 \mathrm{C} 05$.

Key words and phrases. Compact group, metrizable group, Martin's Axiom. 